\title{
Rapid Screening Method for Alpha Thalassemia
}

National Cancer Institute

\section{Source}

National Cancer Institute. Rapid Screening Method for Alpha Thalassemia. NCI

Thesaurus. Code C95541.

Screening techniques for alpha thalassemia that use melting curve analysis of PCR products generated from the alpha globin alleles. 